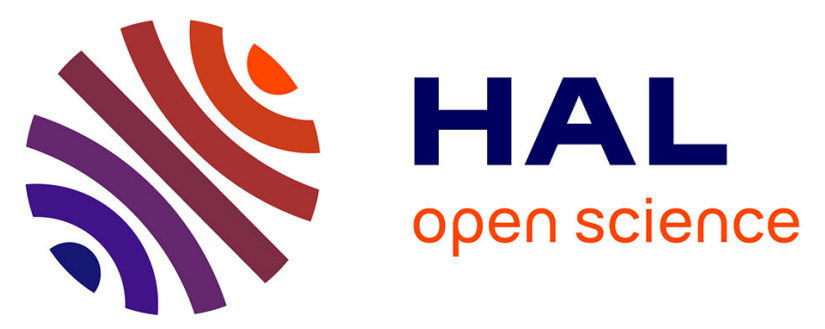

\title{
INFLUENCE DE L'ANCHE ET DE LA TABLE DU BEC SUR LA FRÉQUENCE DE JEU D'UN SYSTÈME À ANCHE SIMPLE
}

Joel Gilbert, X. Meynial, J. Kergomard

\section{- To cite this version:}

Joel Gilbert, X. Meynial, J. Kergomard. INFLUENCE DE L'ANCHE ET DE LA TABLE DU BEC SUR LA FRÉQUENCE DE JEU D'UN SYSTÈME À ANCHE SIMPLE. Journal de Physique Colloques, 1990, 51 (C2), pp.C2-833-C2-836. 10.1051/jphyscol:19902193 . jpa-00230509

HAL Id: jpa-00230509 https://hal.science/jpa-00230509

Submitted on 1 Jan 1990

HAL is a multi-disciplinary open access archive for the deposit and dissemination of scientific research documents, whether they are published or not. The documents may come from teaching and research institutions in France or abroad, or from public or private research centers.
L'archive ouverte pluridisciplinaire HAL, est destinée au dépôt et à la diffusion de documents scientifiques de niveau recherche, publiés ou non, émanant des établissements d'enseignement et de recherche français ou étrangers, des laboratoires publics ou privés. 
COLLOQUE DE PHYSIQUE

Colloque C2, supplément au $\mathrm{n}^{\circ} 2$, Tome 51, Février 1990

ler Congrès Français d'Acoustique 1990

\title{
INFLUENCE DE L'ANCHE ET DE LA TABLE DU BEC SUR LA FREQUENCE DE JEU D'UN SYSTËME À ANCHE SIMPLE
}

\author{
J. GILBERT, X. MEYNIAL et J. KERGOMARD \\ Laboratoire d'Acoustique, CNRS URA 1101, Université du Maine, BP. 535, \\ F-72017 Le Mans Cedex, France
}

\begin{abstract}
Résumé - Nous précisons et proposons une adaptation du modèle classique d'un système anche simple-résonateur (clarinette, saxophone) pour obtenir une évolution de la fréquence de jeu plus réaliste au vu des résultats expérimentaux.

Abstract - We specify and suggest an adaptation of the classical model of single reed like systems (clarinet, saxophone) to obtain a more realistic evolution of playing frequencies as compared with experimental results.
\end{abstract}

1 - INTRODUCTION

Les systèmes à anche simple (clarinettes, saxophones par exemple) sont classiquement modélisés par la combinaison de trols équations à trois inconnues: la pression et la vitesse acoustiques dans le bec, le déplacement à l'extrémité de l'anche. La première équation caractérise l'influence du résonateur par son impédance d'entrée, équation linéalre traduite le plus souvent dans le domaine fréquentlel. La seconde, en première approximation, assimile l'anche à un oscillateur linéaire amorti à un degré de liberté soumis à une différence de pression variable dans le temps (pression dans la cavité buccale moins pression dans le bec), équation linéaire souvent traduite dans le domaine temporel dans la littérature mais qui peut l'être dans le domaine fréquentiel. La trolsième, non linéaire, relie à chaque instant le débit entrant dans l'instrument par l'ouverture anche-table à la différence de pression entre l'intérieur du bec et la cavité buccale de l'instrumentiste (figure 1).

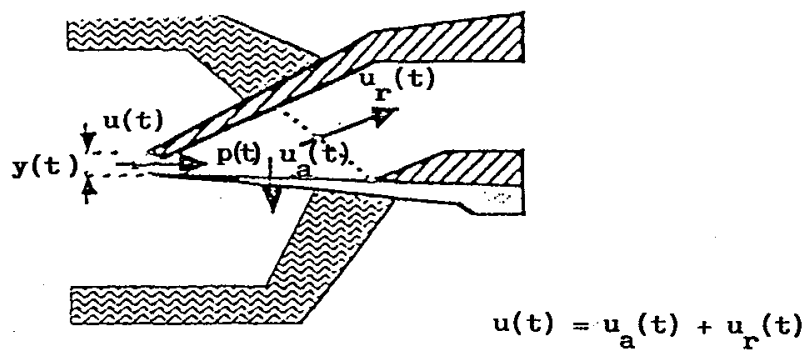

Fig. 1 - Bec de clarinette

$$
\begin{aligned}
& P_{o} \text { : Surpression dans la cavité buccale } \\
& p(t) \text { : Pression acoustique dans le bec } \\
& y(t) \text { : Ouverture anche-table du bec } \\
& u(t) \text { : Débit entrant dans l' Instrument } \\
& u_{r}(t): \text { Débit entrant effectivement dans le résonateur } \\
& u_{a}(t): \text { Débit "pompé" par l'anche }
\end{aligned}
$$




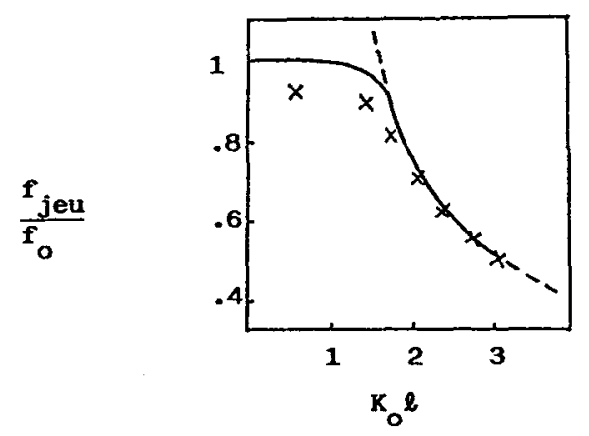

Fig. 2 - Evolution de la fréquence de jeu en fonction de la longueur du résonateur cylindrique

(Figure 2 de $/ 1 /$ )

Théorie (—)

Fréquence de résonance du résonateur cylindrique (------)

Valeurs expérimentales $(X)$

Fréquence de résonance de l'anche : $f_{0}$, $k_{0}$ étant le nombre d'onde correspondant

Il a été montré qu'un tel modèle possède des solutions auto-oscillantes dont les fréquences de jeu peuvent être reliées ses différents paramètres physiques. Une première approche totalement linéaire / $1 /$ met clairement en évidence les deux modes de fonctionnement d'un tel système: le fonctionnement de type clarinette d'une part, la fréquence de jeu. "se cale" sous une fréquence de résonance du tuyau sonore (résonateur cylindrique pour la figure 2), le fonctionnement de type orgue d'autre part, la fréquence de jeu "se cale" sous la première fréquence de résonance de $l$ 'anche. Worman $/ 2 /$ et les approches non-linéalres successives du problème $/ 3,4 /$ montrent que pour un résonateur cylindrique fixé la fréquence de jeu décroit quand la pression d'alimentation crồt à partir de la pression du seuil (figure 3 ).

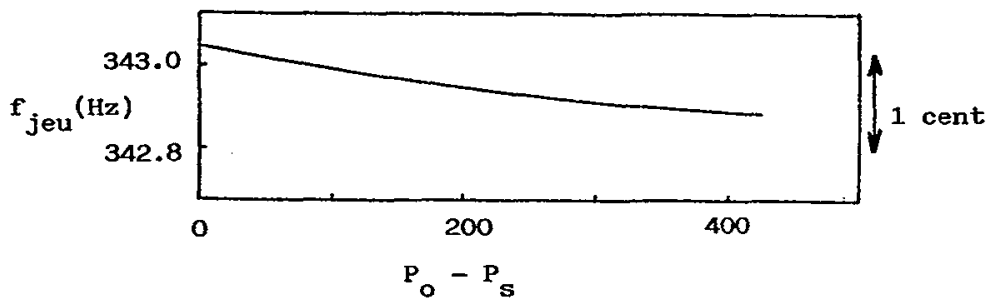

Fig. 3 - Evolution de la fréquence de jeu en fonction de la pression d'alimentation pour un résonateur cylindrique (longueur $24 \mathrm{~cm}$ ) (Courbe donnée par la théorie, figure $18 \mathrm{de} / 2 /$ )

Pression de seuil de l'oscillation: $P_{\text {S }}$ 


\section{2 - INFLUENCE DU DEBIT D'ANCHE SUR LA FREQUENCE DE JEU}

Nous avons pu vérifier expérimentalement (système bec plus résonateur alimentés artificiellement /6/) un écart non négligeable entre la fréquence de jeu du système et la première fréquence de résonance du système passlf bec-réșonance (figure 4). Cet écart fréquentiel peut être expliqué par la présence du terme de débit d'anche: l'osclllation mécanique de l'anche "pompe" une partie du débit entrant dans l'instrument ( $u_{a}$ sur la flgure 1).

$L^{\prime}$ influence de ce terme peut être représentée par un nouveau terme d'impédance $Z_{a} / 3 /$ en parallèle avec l'impédance du résonateur $Z_{r}$ (solt $Z$ l'impédance équivalente). Un premier calcul théorique permet de montrer que $Z_{a}$ provoque un abaissement de la première fréquence $f_{1}$ de l'impédance equivalente d'autant plus important que le cylindre est court (volr la confrontation de la courbe théorique et des valeurs expérimentales figure 4), écart fréquentiel prépondérant devant celui causé par la non-linéarité (figure 3). Notons que 1'absence du terme de débit d'anche dans le modèle de Wilson et Beavers /1/ peut expliquer le décalage fréquentiel entre théorie et expérience sur la figure 2.

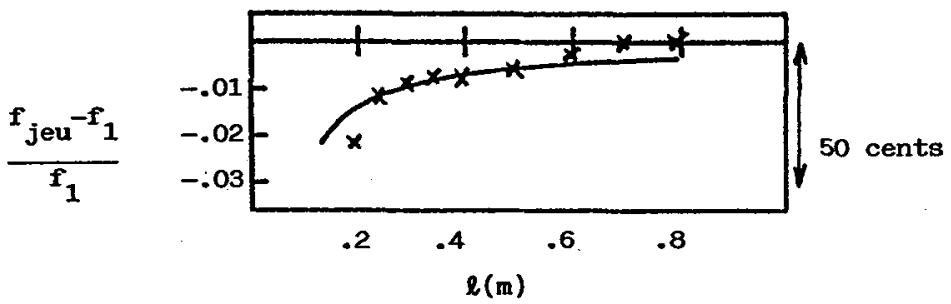

Fig. 4 - Ecart relatif entre la fréquence de jeu et la première fréquence de résonance du résonateur cylindrique en fonction de la longueur de ce dernier

Théorie (-

Valeurs expérimentales $(X)$

Benade $/ \epsilon /$ a introduit la notion de volume équivalent, volume supérieur au volume ġ́ométrique du bec, pour intégrer directement l'écart fréquentiel entre frǽquence de jeu et première fréquence de résonance du corps de l'instrurent lors des mesures d'impédance d'entrée. Ne serait-il pas plus judicieux de laisser à l'entrée de l'instrument un volume égal au volume du bec et de corriger ensuite les résultats expérimentaux ?

\section{3 - UNE PREMIERE PRISE EN COMPTE DE LA COURBURE DE LA TABLE DU BEC}

Nous avo:s pu vérifier qu'une augmentation de la pression d'alimentation, pour un résonateur donné, provoque une légère hausse de la fréquence de jeu $/ 7 /$ (Ehénomène accentué psychologiquement par 1'enrichissement en hauts harmoniques du son résultant). Ce fait expérimental est en contradiction avec le modèle théorique développé jusqu'ici (figure 3). Cette contradiciion (figure 5) est encore plus marquée pour "un résonateur monochroratique" (résonateur constitué de deux tuyaux cylindriques de section ifférente en série $/ 5 /$ comportant une première fréquence de résonance très marquée et une série inharmonique de résonances secondaires). 


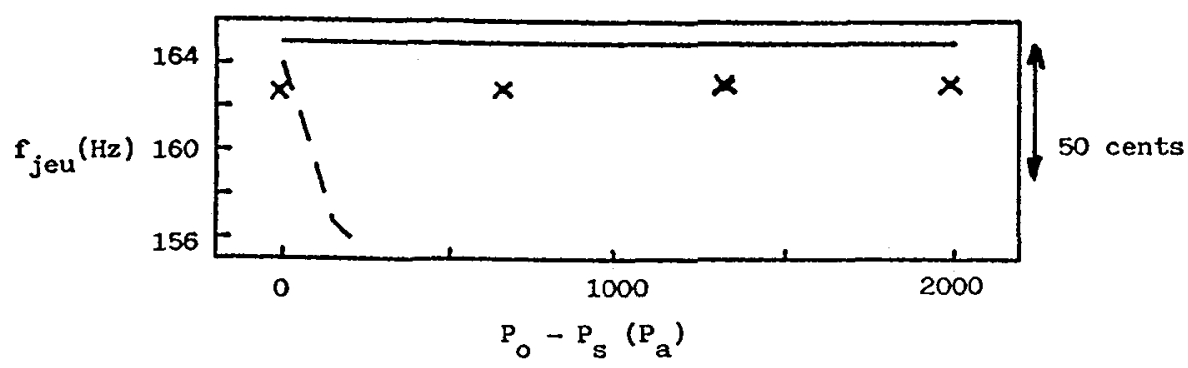

Fig. 5 - Evolution de la fréquence de jeu en fonction de la pression d'alimentation pour un "résonateur monochromatique"

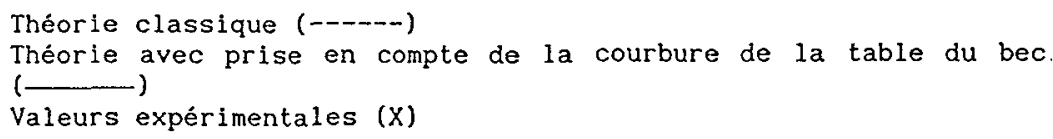

Pour écarter cette difficulté, nous proposons une première prise en compte de la courbure de la table du bec : pour une position de l'anche donnée, il correspond une longueur vibrante de l'anche et donc une fréquence de résonance propre $f_{0}$. Nous avons cherché une dépendance entre $\ell$ et $f_{0}, 101$ du type $f_{0}$ proportionel à $\ell^{-x}$ où $x$ est déterminé empiriquement, intégrant en quelque sorte un grand nombre des propriétés mécaniques de l'anche.

A chaque position de l'extrémité de l'anche, 11 correspond une longueur vibrante, déterminée par la courbure de la table, et donc une fréquence de résonance propre de l'anche. Cette modélisation quasi-statique de l'anche rend non-linéaire la seconde équation du modèle en introduisant une dépendance en $y$ de $f_{0}$. L'équilibrage harmonique $/ 4 /$, méthode numérique d'analyse qui permet le suivi d'un paramètre physique fixé, met en évidence : une évolution plus réaliste de la fréquence de jeu (figure 5), la dissymétrisation de l'allure du signal de déplacement $y(t)$, l'extinction du son vérifiée expérimentalement sur la clarinette alimentée artificiellement $/ 5 /$ pour une pression d'alimentation trop forte.

\section{REFERENCES}

1/ Wilson, A.H., Beavers, G.S., J. Acoust. Soc. Am. 56 (1974), 653. .

/2/ Worman, W.E. , Self-sustained nonlinear oscillations of medium amplitude in clarinet-like systems, Thesis, Case Western Reserve University, Cleveland (1971).

13/ Schumacher, R.T. , Acustica 48 (1981), 71.

/4/ Gilbert, J., Kergomard, J., Ngoya, E., J. Acoust. Soc. Am. 86 (1989), 35.

/5/ Meynial, X., Oscillation d'une anche simple couplée à un résonateur de forme simple, Thèse de Docteur Ingénieur, Université du Maine (1987).

$16 /$ Benade, A.H., Fundamentals of Musical Acoustics", Oxford University Press, N.Y. (1976).

/7/ Bak, N., Domler, P., Acustica 69 (1987), 238. 